\title{
К ВОПРОСУ ОБ АППРОКСИМАЦИИ СКОРОСТЕЙ ВЕТРА НА ЮГО-ВОСТОКЕ ЕВРОПЕЙСКОЙ ТЕРРИТОРИИ РОССИИ ЗАКОНОМ РАСПРЕДЕЛЕНИЯ ВЕЙБУЛЛА-ГУДРИЧА
}

\author{
А.Б. Рыхлов \\ Саратовский государственный университет, \\ кафедра метеорологии и климатологии \\ E-mail: kafmeteo@mail.ru
}

Рассматриваются различные методы оценки параметров распределения Вейбулла-Гудрича для выравнивания скоростей ветра на юго-востоке европейской территории России. Для этой цели рекомендуется использовать методы моментов и наименьших квадратов, минимизирующих объем вычислительных работ. Параметры распределения, оцененные с использование этих параметров, позволяют надежно определять режим скоростей ветра, их средние значения и характеристику изменчивости на юго-востоке европейской территории России.

Ключевые слова: скорость ветра, повторяемость, обеспеченность, режим, распределение, параметр, погрешность.

On the Issue of Wind Speed Approximation by ViebullaGudrich Law on the South-West of European Part of Russian Federation

\section{A.B. Rikhlov}

Different methods of characteristic Viebulla-Gudrich distribution estimation for wind speed leveling on the south-west of European part of Russian Federation are considered. For this purpose is recommended to use moments methods and least-squares method, that minimized the volume of calculating works. Distribution characteristics estimated by these methods allow identifying wind speed conditions, mean values and measures of variability on the south-west of European part of Russian Federation.

Key words: wind speed, periodicity, provision, conditions, distribution, characteristic, error.

Наиболее оптимальным законом для выравнивания скоростного режима ветра, удовлетворяющим критериям Пирсона и Колмогорова, следует признать двухпараметрическое распределение Вейбулла-Гудрича, имеющее вид

$$
F(v)=\exp \left[-\left(\frac{v}{\beta}\right)^{\gamma}\right]
$$

где $v$ - скорость ветра; $\beta$ и $\gamma$ - параметры распределения; $F-$ интегральная вероятность. Для надежного применения этого закона необходимо оценить его параметры $\beta$ и $\gamma$. Оценку параметров данного распределения можно осуществить несколькими методами: графическим, методом наименьших квадратов, максимального правдоподобия, моментов. Ранее, когда средства вычислительной техники были ограничены, наиболее широко использовался предложенный в [1] гра- фический метод, заключающийся в использовании специальной вероятностной клетчатки. При построении такой клетчатки в системе координат $x=\ln v, y=\ln (-\ln F)$ формула (1) преобразуется к виду

$$
y=\gamma x-\gamma \ln \beta,
$$

т.е. в приведенной системе координат функция распределения может быть аппроксимирована прямой. Такие графики очень удобны для статистической экстраполяции данных короткорядных наблюдений, поскольку при этом можно избежать некоторых промежуточных вычислений и даже самого определения параметров $\beta$ и $\gamma$. При необходимости вычисления указанных параметров следует учитывать масштаб, в котором выполнен график. В частности, при использовании рекомендуемых в [1] бланков клетчатки, изготовленных в Ленинградском отделении ТЭП, полученное значение ү оказывается, по расчетам Л.Л. Брагинской [2], заниженным в 1,29 раз.

Достоинством графического метода является его наглядность. Построив такие графики, можно визуально оценить спрямляемость, тесноту связи и разброс точек относительно прямой. Нами такие графики построены для всех метеостанций региона. Все они однозначно подтвердили хорошую спрямляемость. Для иллюстрации этого положения на рис. 1 приведены соответствующие графики выравнивания скоростей распределением Вейбулла по метеостанции Бирючья Коса (Астраханская область) для центральных месяцев сезонов.

Такие графики были построены для более чем 50 метеостанций региона для всех месяцев и года в целом. Все они подтвердили надежную спрямляемость. На рис. 2 они приведены лишь для некоторых станций региона для годовых значений.

Как видно на рис. 2, спрямляющий эффект расположения точек в поле используемых осей координат выражен ярко. Это свидетельствует о том, что выбранный закон распределения скоростей ветра действительно соответствует реальному ветровому режиму на всей рассматриваемой территории. Далее следует оценить параметры $\beta$ и $\gamma$ распределения Вейбулла-Гудрича и получить аналитические выражения, позволяющие воспроизводить вероятность различных скоростей ветра с применением ЭВМ. 

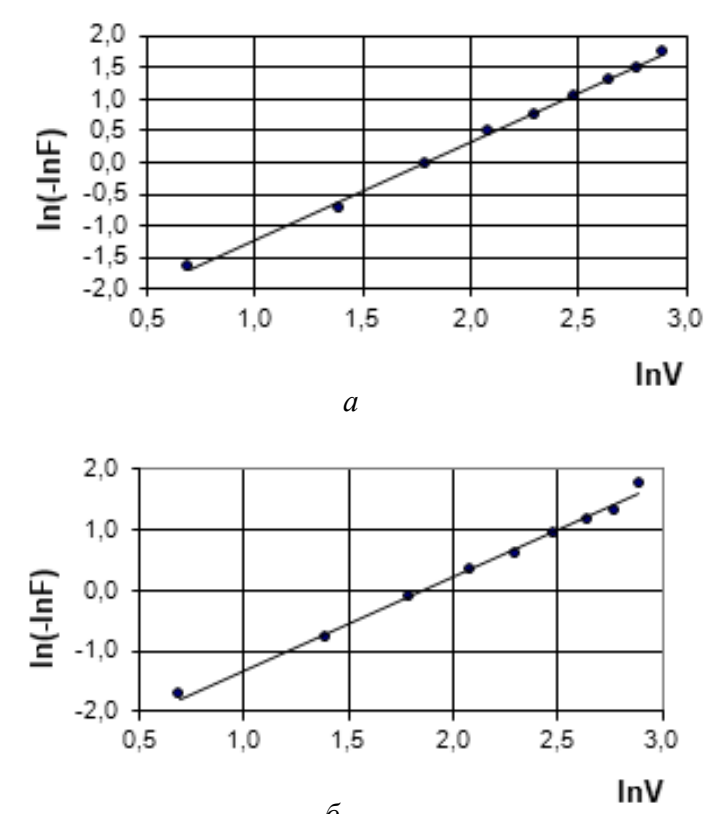
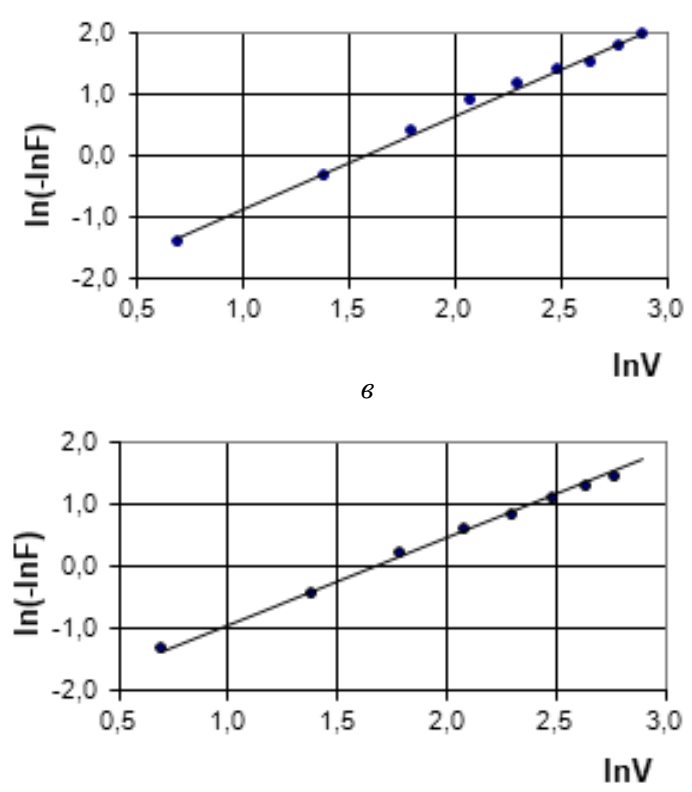

Рис. 1. Фактические обеспеченности скоростей ветра по месяцам для метеостанции Бирючья Коса (Астраханская область): $a$ - январь; $\sigma$ - апрель; 6 - июль; г - октябрь
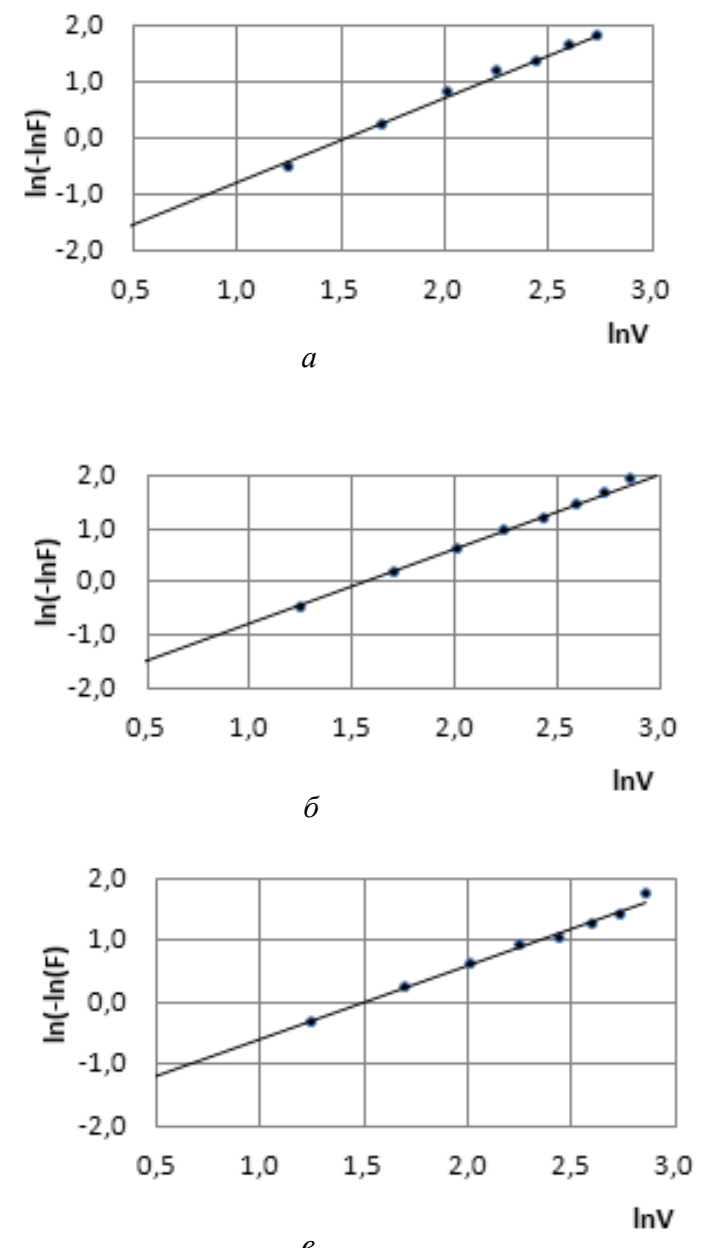
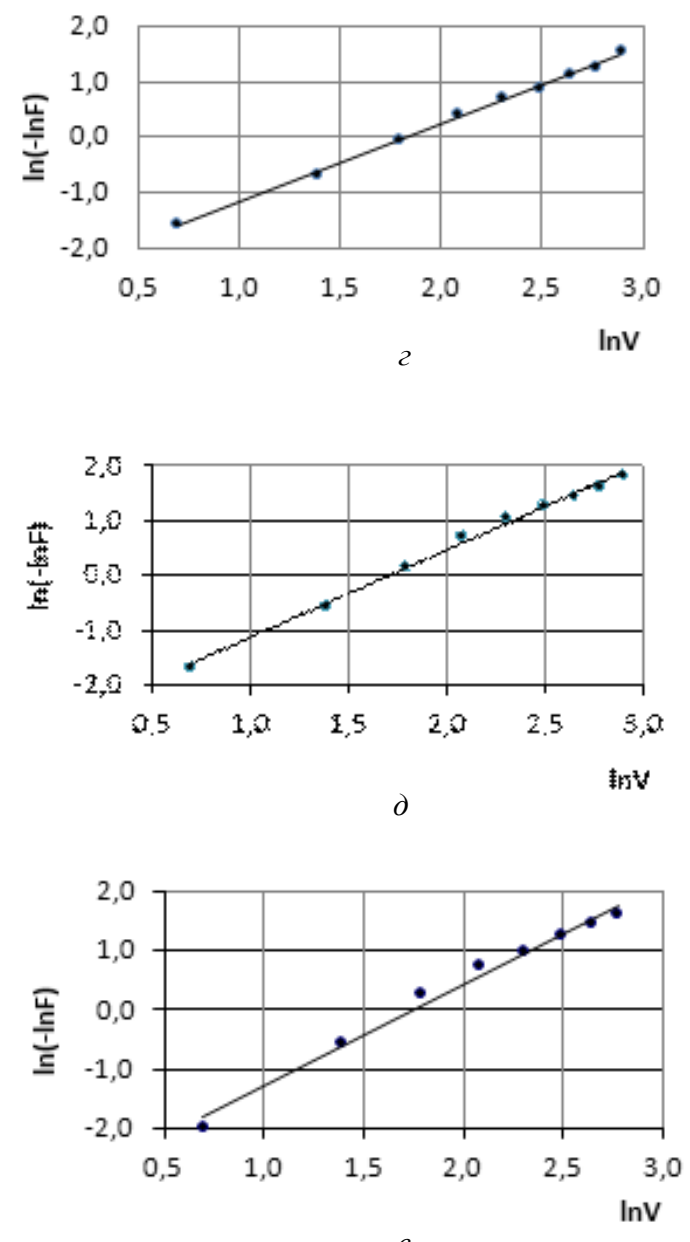

Рис. 2. Прямые распределения скоростей ветра для закона Вейбулла-Гудрича: $a$ - Казань; $\sigma$ - Куйбышев; в- Саратов; 2 - Оренбург; $\partial$ - Волгоград; $e$ - Астрахань 
При использовании графического метода параметр $\gamma$ определяется как угловой коэффициент прямой на графике или тангенс угла ее наклона к оси, на которой отложен $\ln (v)$. Если положить $v=\beta$, то из формулы (1) получим $F(v)=0,368$, поэтому $\beta$ оценивается как скорость ветра, соответствующая данной вероятности. Недостатком графического метода является известный субъективизм, который допускается при проведении прямой по эмпирическим точкам. Учитывая это, С.Д. Кошинский [3] рекомендовал и использовал метод наименьших квадратов, позволяющий по заданным значениям $P(v)$ и $v$ объективно оценить значения $\beta$ и $\gamma$, соответствующие линейной зависимости (2). Они по данным об обеспеченности различной скорости ветра для градаций рассчитываются по формулам:

$$
\begin{gathered}
\gamma=\frac{\sum_{i=1}^{n} \ln \left(-\ln P_{i}\right) \cdot \ln v_{i}-\frac{1}{n} \sum_{i=1}^{n} \ln \left(-\ln P_{i}\right) \cdot \sum_{i=1}^{n} \ln v_{i}}{\sum_{i=1}^{n}\left(\ln v_{i}\right)^{2}-\frac{1}{n}\left(\sum_{i=1}^{n} \ln v_{i}\right)^{2}}, \\
\beta=\exp \left\{-\frac{1}{n \gamma} \sum_{i=1}^{n}\left[\ln \left(-\ln P_{i}\right]-\gamma \cdot \ln v_{i}\right\},\right.
\end{gathered}
$$

где $v_{i}$ и $P_{i}$ - значения скорости ветра и обеспеченность значений скорости ветра, превышающих нижний предел $i$-й градации; $n$ - число градаций, на которые разбит диапазон изменений скорости ветра. В расчетах по формулам (3)-(4) С.Д. Кошинский рекомендует исключать данные крайних градаций, т.е. используются только те значения, при которых

$$
0<P_{i}<1
$$

Это особенно важно, потому что в диапазоне скоростей менее $1 \mathrm{M} / \mathrm{c}$ и более $20 \mathrm{M} / \mathrm{c}$ измерения производятся с большими погрешностями, они способны повлиять на адекватность оценок параметров.

Следует, однако, иметь в виду, что данная процедура, обеспечивая минимальную среднюю квадратическую ошибку построения прямой, по мнению Л.Л. Брагинской [2], вовсе не гарантирует наибольшую точность аппроксимации исходного распределения. Более того, такая процедура из-за нелинейности преобразования координат, вообще говоря, может приводить к смещенности оценок моментов распределения. В силу этого для аппроксимации распределения скоростей ветра было предложено использовать метод максимального правдоподобия, который свободен от указанных недостатков. При использовании этого метода [2] параметры $\beta$ и $\gamma$ находятся путем решения уравнений максимального правдоподобия, которые для сгруппированных данных приводятся к виду

$$
\begin{gathered}
Q=\frac{1}{\gamma}+\sum_{i=1}^{n} P_{i} \cdot \ln v_{i}-\frac{\sum_{i=1}^{n} P_{i} \cdot v_{i}^{\gamma} \cdot \ln v_{i}}{\sum_{i=1}^{n} P_{i} \cdot v_{i}^{\gamma}}=0, \\
\beta=\left(\sum_{i=1}^{n} P_{i} \cdot v_{i}^{\gamma}\right)^{\frac{1}{\gamma}},
\end{gathered}
$$

Трансцендентные уравнения (6)-(7) решаются численно, методом последовательных приближений, что требует весьма трудоемких вычислений при ручных и массовых расчетах. Однако при выполнении расчетов на ЭВМ, что необходимо уже при использовании метода наименьших квадратов, это усложнение расчетов не является принципиальным.

Перечисленные выше методы требуют сведений о распределении скорости ветра во всем диапазоне наблюдаемых значений или, по крайней мере, в большей его части. Учитывая связь между параметрами $\beta$ и $\gamma$ и моментами распределения, для их оценки можно использовать метод моментов, который позволяет получить параметры распределения Вейбулла-Гудрига по любым двум моментам распределения. Следует заметить, что этот метод является исключительно универсальным и эффективным при решении весьма широкого круга как теоретических, так и практических задач в самых различных отраслях знания. В самом деле, можно легко показать, что при использовании распределения Вейбулла-Гудрича для начальных моментов порядка $k$ имеет место соотношение

$$
M_{k}(\beta, \gamma)=\beta^{k} \Gamma\left(1+\frac{k}{\gamma}\right),
$$

где $\Gamma$ - гамма-функция. Отсюда следует, что средняя скорость ветра $v$, среднее квадратическое отклонение $\sigma$ и коэффициент вариации $c_{v}$ могут быть определены по формулам:

$$
\begin{gathered}
\bar{v}=\beta \Gamma\left(1+\frac{1}{\gamma}\right), \\
\sigma^{2}=\beta^{2}\left[\Gamma\left(1+\frac{2}{\gamma}\right)-\Gamma^{2}\left(1+\frac{1}{\gamma}\right)\right], \\
C_{v}=\frac{\sigma}{v}=\frac{\sqrt{\Gamma\left(1+\frac{2}{\gamma}\right)-\Gamma^{2}\left(1+\frac{1}{\gamma}\right)}}{\Gamma\left(1+\frac{1}{\gamma}\right)}:
\end{gathered}
$$

Таким образом, располагая данными о средней скорости ветра и коэффициенте вариации, можно однозначно определить параметры $\beta$ и $\gamma$ распределения Вейбулла-Гудрича. 
Величины $v$ и $\sigma$ с использованием параметров распределения, рассчитанных методом максимального правдоподобия, как отмечала Л.Л. Брагинская [2], хорошо согласуются с результатами непосредственных расчетов. Вместе с тем хорошо согласуются с ними и величины $\beta$ и $\gamma$, полученные методом моментов. Этот очень простой и надежный способ аппроксимации заслуживает особого внимания. Использование же метода наименьших квадратов для определения параметров $\beta$ и $\gamma$, по ее мнению, приводит к завышению расчетных значений средней скорости ветра и ветроэнергетических ресурсов. Среднее квадратическое отклонение, как правило, также оказывается завышенным.

Как показало наше исследование, критика метода наименьших квадратов связана с недоучетом некоторых нюансов климатологической обработки исходных данных. В справочниках по климату $[4,5]$ приведены повторяемости скоростей ветра по градациям. В значительном числе случаев ветер по ветроизмерительному прибору, особенно по флюгеру, в соответствии с методикой производства измерений отсчитывается наблюдателями с точностью лишь до 2 м/с. Это можно обнаружить при построении графика, на котором по абсциссе отложены скорости ветра, по ординате - их повторяемость. В том случае, когда наблюдения не дают точности 1 м/с, линия повторяемости имеет зубчатый вид («пила»). Установлено, что с учетом точности наблюдений для характеристики повторяемости скоростей ветра следует брать интервалы не менее 2 м/с. В результате специальных исследований были установлены наиболее приемлемые границы интервалов: 0-1, 2-3, 4-5, 6-7, 8-9, 10-11, 12-13, 14-15, 16-17, 18-20 м/с, а далее ширина градации увеличивается. Для скоростей ветра более $20 \mathrm{M} / \mathrm{c}$ приняты интервалы через 4 и 6 м/с в соответствии с тем, что у флюгера с тяжелой доской точность наблюдений в диапазоне скоростей от 20 до $28 \mathrm{M} / \mathrm{c}$ составляет 4 м/с, а между 28 и 40 м/с -6 м/с.

По методу моментов параметры распределения $\beta$ и $\gamma$ оцениваются с использованием середины градации, что соответствует точности расчета основных статистических характеристик. Метод наименьших квадратов использует интегральную вероятность (обеспеченность) превышения скорости ветра определенного значения. Переход от повторяемости к обеспеченности скоростей ветра производится путем суммирования повторяемостей от больших значений к меньшим. В связи с принципиальной важностью этого перехода поясним его на примере. Повторяемости скоростей ветра по градациям на метеостанции Анучино за год приведены в табл. 1.

Повторяемость и обеспеченность различных скоростей ветра по метеостанции Анучино

\begin{tabular}{|c|l|c|c|c|c|c|c|c|c|c|c|}
\hline \multirow{2}{*}{$\begin{array}{c}\text { Номер } \\
\text { строки }\end{array}$} & \multicolumn{10}{|c|}{ Параметр } & \multicolumn{10}{c|}{ Значение } \\
\cline { 2 - 14 } & Скорость ветра, м/с & $0-1$ & $2-3$ & $4-5$ & $6-7$ & $8-9$ & $10-11$ & $12-13$ & $14-15$ & $16-17$ & $18-20$ \\
\hline 1 & Номер градации & 1 & 2 & 3 & 4 & 5 & 6 & 7 & 8 & 9 & 10 \\
\hline 2 & Повторяемость & 0,184 & 0,249 & 0,245 & 0,159 & 0,088 & 0,032 & 0,023 & 0,01 & 0,008 & 0,002 \\
\hline 3 & Скорость ветра, м/с & $\geq 0$ & $\geq 2$ & $\geq 4$ & $\geq 6$ & $\geq 8$ & $\geq 10$ & $\geq 12$ & $\geq 14$ & $\geq 16$ & $\geq 18$ \\
\hline 4 & Обеспеченность & 1,0 & 0,816 & 0,567 & 0,322 & 0,163 & 0,075 & 0,043 & 0,02 & 0,01 & 0,002 \\
\hline
\end{tabular}

Измерение скорости ветра на метеостанциях производится с точностью до целых значений. Поэтому вероятность последней градации (в данном примере 18-20 м/с), как правило, соответствует вероятности того, что скорость ветра будет более 18 м/с (строка 4, градация 10). Суммируя градации 10 и 9, получаем обеспеченность скорости ветра более $16 \mathrm{~m} / \mathrm{c}$ и т.д. Нанося эти скорости ветра и их интегральные вероятности на специальные графики в билогарифмической системе координат, графическим методом, методом моментов или наименьших квадратов можно рассчитать параметры $\beta$ и $\gamma$ распределения Вейбулла-Гудрича. Их оценки для дискретных значений скоростей ветра приведены в табл. 2. Из нее следует, что методом наименьших квадратов параметры $\beta$ и $\gamma$ распределения Вейбулла-Гудрига заметно завышены во все месяцы и за год в целом. Величина завышения, примерно того же уровня, что и у Л.Л. Брагинской [3], которую она объясняла нелинейностью преобразований.

Таблица 2

Параметры распределения Вейбулла-Гудрича, оцененные различными методами для дискретных значений скорости ветра

\begin{tabular}{|c|c|c|c|c|c|c|c|c|c|c|c|c|}
\hline \multirow{3}{*}{ Месяц } & \multicolumn{4}{|c|}{ Анучино } & \multicolumn{4}{|c|}{ Заметчино } & \multicolumn{4}{|c|}{ Пенза } \\
\hline & \multicolumn{2}{|c|}{$\beta$} & \multicolumn{2}{|c|}{$\gamma$} & \multicolumn{2}{|c|}{$\beta$} & \multicolumn{2}{|c|}{$\gamma$} & \multicolumn{2}{|c|}{$\beta$} & \multicolumn{2}{|c|}{$\gamma$} \\
\hline & 1 & 2 & 1 & 2 & 1 & 2 & 1 & 2 & 1 & 2 & 1 & 2 \\
\hline I & 1,40 & 1,53 & 5,61 & 6,23 & 1,28 & 1,41 & 4,96 & 5,62 & 1,44 & 1,64 & 5,25 & 6,05 \\
\hline II & 1,39 & 1,49 & 5,91 & 6,54 & 1,28 & 1,37 & 5,19 & 5,84 & 1,45 & 1,64 & 5,09 & 5,89 \\
\hline
\end{tabular}


Окончание табл. 2

\begin{tabular}{|c|c|c|c|c|c|c|c|c|c|c|c|c|}
\hline \multirow{3}{*}{ Месяц } & \multicolumn{4}{|c|}{ Анучино } & \multicolumn{4}{|c|}{ Заметчино } & \multicolumn{4}{|c|}{ Пенза } \\
\hline & \multicolumn{2}{|c|}{$\beta$} & \multicolumn{2}{|c|}{$\gamma$} & \multicolumn{2}{|c|}{$\beta$} & \multicolumn{2}{|c|}{$\gamma$} & \multicolumn{2}{|c|}{$\beta$} & \multicolumn{2}{|c|}{$\gamma$} \\
\hline & 1 & 2 & 1 & 2 & 1 & 2 & 1 & 2 & 1 & 2 & 1 & 2 \\
\hline III & 1,42 & 1,52 & 5,90 & 6,53 & 1,30 & 1,43 & 5,02 & 5,71 & 1,44 & 1,64 & 5,31 & 6,17 \\
\hline IV & 1,42 & 1,58 & 4,86 & 5,45 & 1,34 & 1,52 & 4,42 & 5,07 & 1,44 & 1,63 & 4,88 & 5,68 \\
\hline $\mathrm{V}$ & 1,42 & 1,58 & 4,87 & 5,51 & 1,34 & 1,50 & 4,53 & 5,25 & 1,42 & 1,54 & 4,70 & 5,53 \\
\hline VI & 1,43 & 1,65 & 4,25 & 4,82 & 1,34 & 1,50 & 3,85 & 4,50 & 1,35 & 1,53 & 4,09 & 4,86 \\
\hline VII & 1,36 & 1,57 & 3,77 & 4,35 & 1,23 & 1,40 & 3,24 & 3,85 & 1,38 & 1,55 & 3,79 & 4,50 \\
\hline VIII & 1,38 & 1,54 & 4,00 & 4,62 & 1,17 & 1,30 & 3,18 & 3,79 & 1,40 & 1,58 & 3,83 & 4,52 \\
\hline IX & 1,47 & 1,65 & 4,59 & 5,23 & 1,26 & 1,43 & 3,82 & 4,48 & 1,58 & 1,76 & 4,84 & 5,62 \\
\hline $\mathrm{X}$ & 1,44 & 1,59 & 5,04 & 5,73 & 1,30 & 1,45 & 4,22 & 4,90 & 1,48 & 1,67 & 5,34 & 6,13 \\
\hline $\mathrm{XI}$ & 1,42 & 1,57 & 5,31 & 6,03 & 1,36 & 1,52 & 4,68 & 5,38 & 1,44 & 1,65 & 4,94 & 5,79 \\
\hline XII & 1,46 & 1,62 & 5,81 & 6,45 & 1,40 & 1,52 & 5,27 & 5,93 & 1,43 & 1,64 & 5,27 & 6,11 \\
\hline Год & 1,38 & 1,52 & 4,97 & 5,65 & 1,27 & 1,40 & 4,37 & 5,04 & 1,40 & 1,59 & 4,78 & 5,58 \\
\hline
\end{tabular}

Примечание. 1 - метод моментов, 2 - метод наименьших квадратов.

Однако в результате нашего исследования удалось обнаружить, что при допущении не дискретного, а непрерывного изменения значений скоростей ветра, параметры строки 4 табл. 1 следует заменить на $\geq 0,0, \geq 1,5, \geq 3,5, \geq 5,5, \geq 7,5, \geq 9,5, \geq 11,5$, $\geq 13,5, \geq 15,5, \geq 17,5$. Это соответствует значениям середины промежутка между градациями. Построение кривых обеспеченностей в билогарифмической системе координат по указанным значениям и оценка параметров распределения Вейбулла-Гудрича $\beta$ и $\gamma$ методом наименьших квадратов в этих условиях позволяют получить принципиально новый результат. Расхождения полученных пара- метров, как методом наименьших квадратов, так и методом моментов, находятся на уровне сотых долей единицы и не превышают случайных счетных ошибок, например округления (табл. 3). Это позволяет сделать важный методический вывод: параметры распределения Вейбулла-Гудрича $\beta$ и $\gamma$ без ущерба для точности получаемых характеристик можно рассчитывать как методом моментов, так и методом наименьших квадратов. Для подтверждения данного вывода приведем оценку параметров $\beta$ и $\gamma$, средних значений и средних квадратических отклонений скоростей ветра с использованием формул (3)-(4) и (9)-(10).

Таблиия 3 Параметры распределения Вейбулла-Гудрича, оцененные различными методами для непрерывных скоростей ветра

\begin{tabular}{|c|c|c|c|c|c|c|c|c|c|c|c|c|}
\hline \multirow{3}{*}{ Месяц } & \multicolumn{4}{|c|}{ Анучино } & \multicolumn{4}{|c|}{ Заметчино } & \multicolumn{4}{|c|}{ Пенза } \\
\hline & \multicolumn{2}{|c|}{$\gamma$} & \multicolumn{2}{|c|}{$\beta$} & \multicolumn{2}{|c|}{$\gamma$} & \multicolumn{2}{|c|}{$\beta$} & \multicolumn{2}{|c|}{$\gamma$} & \multicolumn{2}{|c|}{$\beta$} \\
\hline & 1 & 2 & 1 & 2 & 1 & 2 & 1 & 2 & 1 & 2 & 1 & 2 \\
\hline I & 1,40 & 1,39 & 4,98 & 5,00 & 1,28 & 1,26 & 4,98 & 5,00 & 1,42 & 1,44 & 5,35 & 5,38 \\
\hline II & 1,39 & 1,33 & 5,19 & 5,17 & 1,28 & 1,26 & 5,19 & 5,17 & 1,44 & 1,47 & 5,19 & 5,21 \\
\hline III & 1,42 & 1,36 & 5,02 & 5,01 & 1,30 & 1,28 & 5,02 & 5,01 & 1,38 & 1,40 & 5,49 & 5,50 \\
\hline IV & 1,42 & 1,41 & 4,48 & 4,50 & 1,34 & 1,33 & 4,48 & 4,50 & 1,42 & 1,44 & 4,95 & 5,00 \\
\hline $\mathrm{V}$ & 1,42 & 1,42 & 4,58 & 4,60 & 1,34 & 1,35 & 4,58 & 4,60 & 1,31 & 1,34 & 4,78 & 4,80 \\
\hline VI & 1,43 & 1,48 & 3,85 & 3,86 & 1,34 & 1,35 & 3,85 & 3,86 & 1,34 & 1,36 & 4,09 & 4,11 \\
\hline VII & 1,36 & 1,40 & 3,24 & 3,26 & 1,23 & 1,20 & 3,24 & 3,28 & 1,38 & 1,39 & 3,80 & 3,81 \\
\hline VIII & 1,38 & 1,38 & 3,18 & 3,20 & 1,17 & 1,16 & 3,18 & 3,20 & 1,40 & 1,40 & 3,83 & 3,83 \\
\hline IX & 1,47 & 1,48 & 3,83 & 3,85 & 1,28 & 1,28 & 3,83 & 3,85 & 1,58 & 1,58 & 4,94 & 4,95 \\
\hline$X$ & 1,44 & 1,43 & 4,22 & 4,26 & 1,30 & 1,26 & 4,22 & 4,26 & 1,48 & 1,50 & 5,33 & 5,36 \\
\hline XI & 1,42 & 1,41 & 4,68 & 4,70 & 1,36 & 1,36 & 4,68 & 4,70 & 1,46 & 1,46 & 4,98 & 5,00 \\
\hline XII & 1,46 & 1,45 & 5,27 & 5,26 & 1,40 & 1,36 & 5,27 & 5,26 & 1,46 & 1,45 & 5,37 & 5,37 \\
\hline Год & 1,38 & 1,37 & 4,36 & 4,38 & 1,27 & 1,26 & 4,36 & 4,38 & 1,42 & 1,42 & 4,78 & 4,80 \\
\hline
\end{tabular}

Примечание. 1 - метод моментов, 2 - метод наименьших квадратов. 
Для ряда станций юго-востока ЕТР, находящихся в различных климатических зонах, результаты расчетов при использовании различных способов аппроксимации распределения скорости ветра законом Вейбулла-Гудрича представлены в табл. 4. Естественно, что значения $\bar{v}$, полученные прямым методом (метод 2 , табл. 1), практически совпадают со значениями, взятыми из [4] (метод 1, табл. 4). Некоторое расхождение, находящееся, как правило, в пределах 0,1 м/с, объясняется тем, что при расчете прямым методом использовались значения, рассчитанные не непосредственно из всей совокупности данных, как значения, приведенные в табл. 3 работы [4], а путем использования сгруппированных данных. Представляется, что значения $\bar{v}$ и $\sigma$, полученные непосредственно по сгруппированным данным, являются близкими к действительности. Для сравнения приведена средняя многолетняя скорость ветра из [4].

Таблица 4

Средние скорости ветра, м/с, рассчитанные различными методами

\begin{tabular}{|c|c|c|c|c|c|c|c|c|c|c|c|c|}
\hline \multirow{2}{*}{ Месяц } & \multicolumn{4}{|c|}{ Анучино } & \multicolumn{4}{|c|}{ Заметчино } & \multicolumn{5}{|c|}{ Пенза } \\
\cline { 2 - 14 } & 1 & 2 & 3 & 4 & 1 & 2 & 3 & 4 & 1 & 2 & 3 & 4 \\
\hline I & 5,1 & 5,1 & 5,1 & 5,1 & 4,6 & 4,6 & 4,6 & 4,6 & 4,8 & 4,8 & 4,8 & 4,9 \\
\hline II & 5,4 & 5,4 & 5,4 & 5,4 & 4,8 & 4,8 & 4,8 & 4,8 & 4,6 & 4,6 & 4,6 & 4,7 \\
\hline III & 5,4 & 5,4 & 5,4 & 5,2 & 4,6 & 4,6 & 4,6 & 4,6 & 4,8 & 4,8 & 4,8 & 4,9 \\
\hline IV & 4,4 & 4,4 & 4,4 & 4,4 & 4,1 & 4,1 & 4,1 & 4,1 & 4,4 & 4,4 & 4,4 & 4,5 \\
\hline V & 4,4 & 4,4 & 4,4 & 4,4 & 4,2 & 4,2 & 4,2 & 4,2 & 4,3 & 4,3 & 4,3 & 4,4 \\
\hline VI & 3,9 & 3,9 & 3,9 & 3,8 & 3,5 & 3,5 & 3,5 & 3,5 & 3,8 & 3,8 & 3,8 & 3,9 \\
\hline VII & 3,5 & 3,5 & 3,5 & 3,4 & 3,0 & 3,0 & 3,0 & 3,1 & 3,5 & 3,5 & 3,5 & 3,5 \\
\hline VIII & 3,6 & 3,6 & 3,6 & 3,6 & 3,0 & 3,0 & 3,0 & 3,0 & 3,5 & 3,5 & 3,5 & 3,6 \\
\hline IX & 4,2 & 4,2 & 4,2 & 4,1 & 3,6 & 3,6 & 3,6 & 3,6 & 4,3 & 4,3 & 4,3 & 4,4 \\
\hline X & 4,6 & 4,6 & 4,6 & 4,6 & 3,9 & 3,9 & 3,9 & 4,0 & 4,8 & 4,8 & 4,8 & 4,9 \\
\hline XI & 4,8 & 4,8 & 4,8 & 4,9 & 4,3 & 4,3 & 4,3 & 4,3 & 4,5 & 4,5 & 4,5 & 4,6 \\
\hline XII & 5,3 & 5,3 & 5,3 & 5,2 & 4,8 & 4,8 & 4,8 & 4,8 & 4,8 & 4,8 & 4,8 & 4,9 \\
\hline Год & 4,5 & 4,5 & 4,5 & 4,5 & 4,0 & 4,0 & 4,0 & 4,1 & 4,3 & 4,3 & 4,3 & 4,4 \\
\hline
\end{tabular}

Примечание. 1 - данные из «Справочника по климату СССР», 2 - данные, рассчитанные по повторяемости градаций, 3 - методом моментов, 4 - методом наименьших квадратов.

Расчеты показали, что при предложенном нами способе использования метода наименьших квадратов, значения средних скоростей ветра практически идентичны значениям, полученным как прямыми, так и косвенными (метод моментов) расчетами (см. табл. 4). Различия связаны лишь с точностью округления результатов, так как они имеют разные знаки в пределах $0,1 \mathrm{~m} / \mathrm{c}$. Такой же результат показали расчеты средних квадрати- ческих отклонений и коэффициентов вариации.

В задачу данной работы не входит подробный анализ параметров распределения. Отметим лишь, что параметр $\beta$ близок к значению средней скорости ветра, его годовой ход соответствует годовому ходу скорости ветра. Параметр $\gamma$ меняется на рассматриваемой территории от 1,0 до 1,7 , в большинстве пунктов он не имеет выраженного годового хода (табл. 5).

Таблица 5

Параметры распределения Вейбулла-Гудрича $\gamma$ и $\beta$ для некоторых метеостанций региона

\begin{tabular}{|l|c|c|c|c|c|c|c|c|c|c|c|c|c|c|}
\hline \multicolumn{1}{|c|}{ Станция } & & $\mathrm{I}$ & $\mathrm{II}$ & $\mathrm{III}$ & $\mathrm{IV}$ & $\mathrm{V}$ & $\mathrm{VI}$ & $\mathrm{VII}$ & $\mathrm{VIII}$ & $\mathrm{IX}$ & $\mathrm{X}$ & $\mathrm{XI}$ & $\mathrm{XII}$ & Год \\
\hline \multirow{2}{*}{ Казань } & $\gamma$ & 1,47 & 1,48 & 1,46 & 1,49 & 1,66 & 1,58 & 1,56 & 1,48 & 1,59 & 1,70 & 1,57 & 1,52 & 1,51 \\
\cline { 2 - 15 } & $\beta$ & 5,29 & 4,87 & 5,15 & 4,41 & 4,83 & 4,05 & 3,84 & 3,83 & 4,37 & 5,10 & 5,02 & 5,17 & 4,65 \\
\hline \multirow{2}{*}{ Саратов } & $\beta$ & 1,11 & 1,14 & 1,16 & 1,14 & 1,23 & 1,22 & 1,29 & 1,16 & 1,23 & 1,19 & 1,13 & 1,17 & 1,16 \\
\cline { 2 - 14 } & $\beta$ & 4,63 & 4,93 & 5,02 & 4,56 & 4,55 & 3,99 & 3,95 & 3,59 & 4,14 & 4,66 & 4,48 & 5,19 & 4,46 \\
\hline \multirow{2}{*}{ Эльтон } & $\gamma$ & 1,48 & 1,50 & 1,54 & 1,39 & 1,45 & 1,51 & 1,48 & 1,42 & 1,31 & 1,38 & 1,51 & 1,52 & 1,45 \\
\cline { 2 - 13 } & $\beta$ & 4,56 & 4,96 & 4,75 & 4,33 & 4,38 & 4,16 & 3,98 & 3,76 & 3,66 & 4,14 & 4,39 & 4,57 & 4,30 \\
\hline \multirow{2}{*}{ Оренбург } & $\gamma$ & 1,17 & 1,17 & 1,14 & 1,17 & 1,20 & 1,32 & 1,27 & 1,13 & 1,12 & 1,16 & 1,17 & 1,18 & 1,14 \\
\cline { 2 - 13 } & $\beta$ & 4,86 & 5,66 & 5,38 & 4,68 & 5,04 & 4,17 & 3,86 & 3,55 & 3,51 & 4,38 & 4,48 & 5,17 & 4,51 \\
\hline \multirow{2}{*}{$\begin{array}{l}\text { Астрахань, } \\
\text { ГМО }\end{array}$} & $\gamma$ & 1,66 & 1,62 & 1,54 & 1,45 & 1,57 & 1,56 & 1,62 & 1,50 & 1,42 & 1,46 & 1,48 & 1,62 & 1,50 \\
\cline { 2 - 12 } & $\beta$ & 5,10 & 5,24 & 5,57 & 5,51 & 5,21 & 4,81 & 4,17 & 4,00 & 4,13 & 4,42 & 4,94 & 5,14 & 4,84 \\
\hline
\end{tabular}


Итак, в результате проведенного исследования установлена принципиальная возможность применения распределения Вейбулла-Гудрича к выравниванию скоростей ветра на уровне ветроизмерительного прибора. Это открывает новые возможности для обработки информации о ветровом режиме с применением средств вычислительной техники для решения широкого круга научных и практических задач. К ним можно отнести оценку ветрового напора на здания и сооружения, застойных состояний атмосферы, потенциальных и утилизируемых ветроэнергетических ресурсов и их распределения по территории. Для оценки параметров этого распределения рекомендуется использовать методы моментов и наименьших квадратов, минимизирующих объем вычислительных работ. Параметры распределения, оцененные с использование этих параметров, позволяют надежно определять средние скорости ветра и характеристики изменчивости.

\section{Библиографический список}

1. Анапольская Л.Е. Режим скоростей ветра на территории СССР. Л., 1961. 199 с.

2. Брагинская Л.Л., Каган Р.Л. К вопросу об аппроксимации распределения скоростей ветра // Тр. ГГО. 1982. Вып. 447. С. 49-57.

3. Кошинский С.Д. Из опыта расчета некоторых статистических характеристик ветра и параметров функции распределения вида $f(x)=\exp \left[-\left(\frac{v}{\beta}\right)^{\gamma}\right]$ на электронновычислительных машинах // Тр. НР ГМЦ СССР. 1969.

Вып. 2. С. 44-53.

4. Справочник по климату СССР: В 5 ч. Ч. 3, вып. 12-13. Л., 1967.

5. Научно-прикладной справочник по климату СССР. Л., 1988. Сер. 3. Вып. 12-13.

УДК 91(075.8)+929

\section{САРАТОВСКИЙ МЕЖРЕГИОНАЛЬНЫЙ ЦЕНТР КОСМИЧЕСКОГО МОНИТОРИНГА КАК ФАКТОР ИНТЕГРАЦИИ НАУКИ, ОБРАЗОВАНИЯ И БИЗНЕСА}

\section{В.3. Макаров, А.Н. Чумаченко}

Саратовский государственный университет, кафедра физической географии и ландшафтной экологии, ${ }^{1}$ кафедра геоморфологии и геоэкологии E-mail: geogr@sgu.ru

В статье рассмотрены результаты деятельности научно-внедренческого образовательного центра геоинформационных технологий (ГИС-центр). Кратко освещены выполненные в ГИС-центре проекты по внедрению ГИС-методологии в научно-производственные и производственные организации Поволжья и намечены основные направления работы новой инновационной структуры СГУ - межрегионального центра космического мониторинга.

Ключевые слова: инновационная деятельность, геоинформатика, ГИС-технологии, комплексный территориальный анализ и региональное планирование, дистанционное зондирование, космические снимки.

Saratov Interregional Centre of Space Monitoring as a Factor of Science, Education and Business Integration

\section{V.Z. Makarov, A.N. Chumachenko}

The article represents results of scientific-innovation educational centre of geoinformational technologies (GIS-centre) activity. Also briefly reports projects realized by GIS-centre concerning of GIS methodology implementation to Povolzhie' scientific-production and production organizations and lay down general working trends of the Saratov State University new innovation organization - the interregional centre of space monitoring.

Key words: innovation activity, geoinformatic, GIS-technology, complex territorial analysis and regional planning, remote sensing, space images, spacephotomaps.

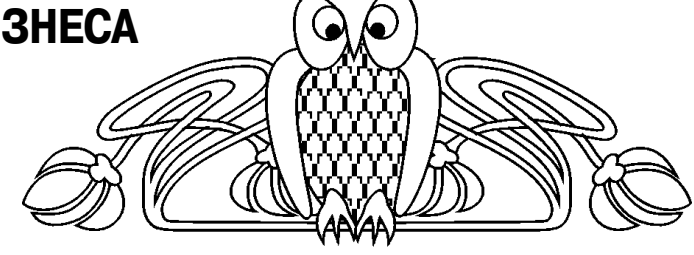

\section{Введение}

Альтернативы инновационному развитию страны на сегодняшний день нет. Этот широко используемый тезис не оспаривается. В то же время призывы к инновационному развитию так и останутся призывами, если среди прочего не заработают инновационные университетские центры. Нет в нашей стране других структур, в которых могли бы быть реализованы задачи не только генерирования новых идей, разработки прорывных технологий, но, одновременно, и задачи подготовки высококлассных специалистов, способных подхватить эти идеи, внедрить новые технологии в нашу жизнь. Как показывает опыт, отсутствие хотя бы одной из составляющих инновационного развития не просто тормозит процесс развития, a, по сути, останавливает его. Разумеется, указанные условия являются необходимыми, но далеко не достаточными для поступательного движения. В частности, нужно, чтобы университетские научнообразовательные центры выступали фокусом интеграции приоритетных научных направлений, современных образовательных парадигм и запросов бизнеса.

В Саратовском государственном университете создан и более 3 лет работает научно- 